\title{
Images of the month: Cavernous sinus venous thrombosis secondary to Streptococcus milleri maxillary sinusitis: An unusual cause of diplopia and headache
}

\author{
Authors: Vanesa Anton-Vazquez, ${ }^{\mathrm{A}}$ Robin Dru, ${ }^{\mathrm{B}}$ Philip Rich, ${ }^{\mathrm{C}}$ Mauricio Arias ${ }^{\mathrm{D}}$ and Derek Macallan ${ }^{\mathrm{E}}$
}

\begin{abstract}
Cavernous sinus venous thrombosis is an uncommon condition associated with high mortality rates if not recognised early. Symptoms include headache, visual loss, ophthalmoplegia, altered consciousness, proptosis and periorbital oedema. High-quality imaging is critical in early diagnosis and successful management. Primary infection (such as sinusitis) and possible complications (including meningitis) should be considered as potential aetiologies of cavernous sinus venous thrombosis, especially in those with a preceding history of localised infection. We present a case of a 50-yearold man with a bilateral cavernous sinus venous thrombosis with associated meningitis caused by Streptococcus milleri, secondary to maxillary sinusitis and otomastoiditis. He was successfully treated with antimicrobial treatment, surgical drainage and anticoagulation.
\end{abstract}

KEYWORDS: Cavernous sinus venous thrombosis, diplopia, headache, sinusitis, Streptococcus milleri

DOI: $10.7861 /$ clinmed.2020-0689

\section{Case presentation}

A 50-year-old man, with a history of asthma on corticosteroid inhalers, presented with a 2-week history of diplopia and headache. He had been reviewed 3 weeks earlier due to right ear pain and was diagnosed with otitis externa for which he received a 1-week course of oral clarithromycin. A week after which, he developed progressively worsening diplopia and headache.

On examination, at the time of admission, he was alert and orientated with a Glasgow coma score of 15/15. He was afebrile

Authors: A registrar in infectious diseases and medical microbiology, St George's University Hospitals NHS Foundation Trust, London, UK; ${ }^{\text {B }}$ clinical teaching fellow in emergency medicine, St George's University Hospitals NHS Foundation Trust, London, UK;

C consultant in neuroradiology, St George's University Hospitals NHS Foundation Trust, London, UK; ${ }^{D}$ consultant in medical microbiology and infectious diseases, St George's University Hospitals NHS Foundation Trust, London, UK; ${ }^{\mathrm{E}}$ consultant in infectious diseases, St George's University Hospitals NHS Foundation Trust, London, UK and St George's, University of London, London, UK (body temperature of $36.5^{\circ} \mathrm{C}$ ) but hypertensive $(200 / 100 \mathrm{mmHg}$ ); observations were otherwise unremarkable. He had marked diplopia, with bilateral VI cranial nerve palsies evident on examination. Hearing in the right ear was reduced and a purulent discharge from the ear was noted. There was mild nuchal rigidity and photophobia. Blood tests revealed $25.7 \times 10^{9} / \mathrm{L}$ leucocytes, $21.7 \times 10^{9} / \mathrm{L}$ neutrophils and a mildly raised C-reactive protein $(37 \mathrm{~g} / \mathrm{dL})$.

Imaging revealed extensive sinusitis with opacification of right maxillary antrum (Fig 1) and bilateral otomastoiditis (not shown). The striking feature in this case however was the finding on magnetic resonance imaging (MRI) of the brain (Fig 2) that revealed the diagnosis.

MRI of the brain demonstrated bilateral cavernous sinus thrombosis with enhanced and thickened lateral dural walls due to inflammation. Lumbar puncture showed raised opening pressure of $32 \mathrm{cmH}_{2} \mathrm{O}$. Cerebrospinal fluid had 1,400 white cells $(65 \%$

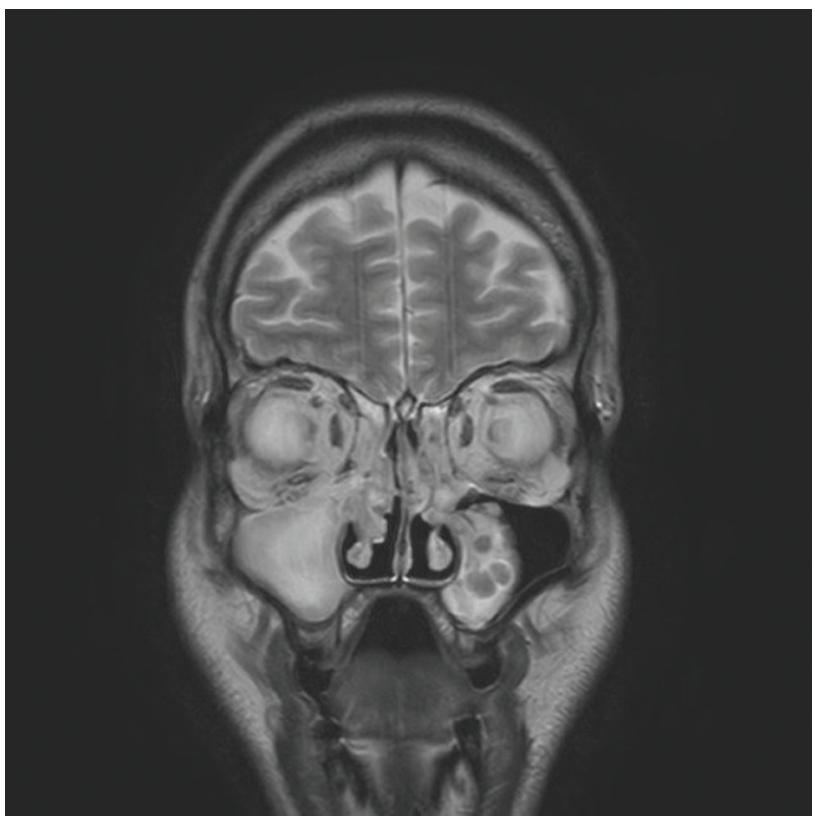

Fig 1. Coronal T2 weighted magnetic resonance imaging revealing generalised paranasal sinus mucosal thickening and small nasal polyps and opacification of right maxillary antrum due to sinusitis. 

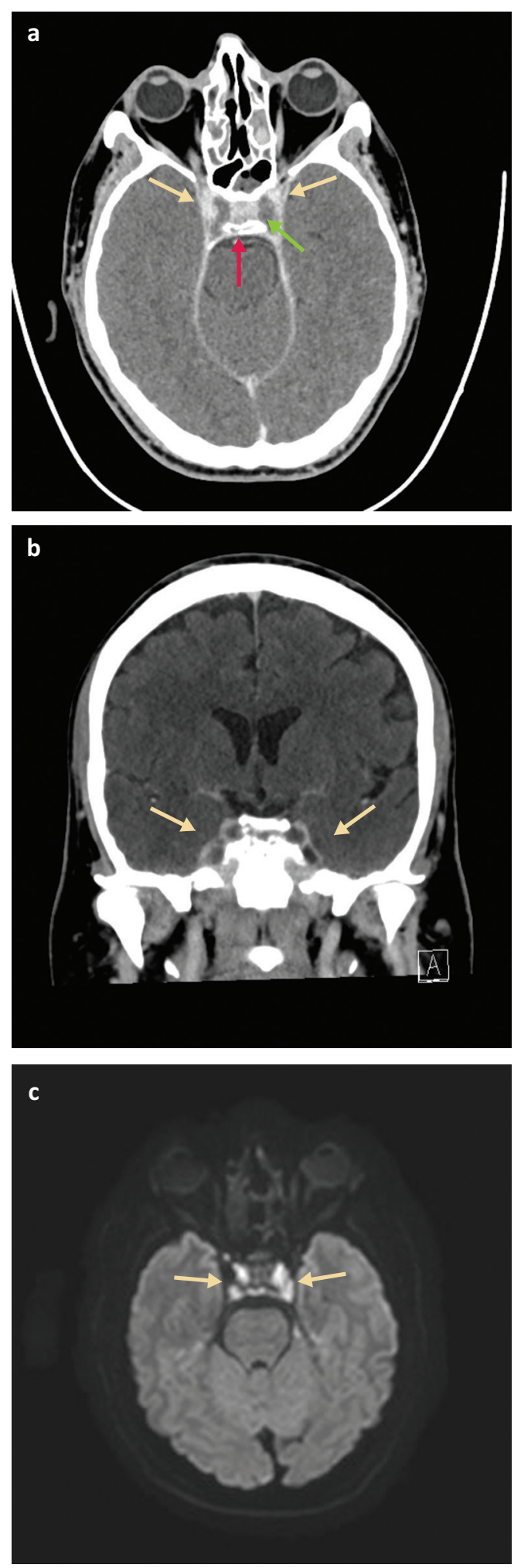

Fig 2. a) Contrast enhanced axial computed tomography. Both cavernous sinuses are expanded and do not enhance centrally due to thrombus (green arrow). Normal enhancement of the internal carotid arteries (yellow arrows) and pituitary gland (red arrow). b) Contrast enhanced coronal computed tomography. Both cavernous sinuses (yellow arrows) are expanded and do not enhance centrally due to thrombus. The lateral dural walls are thickened due to inflammation and enhance avidly. c) Diffusion weighted axial magnetic resonance imaging. Infected thrombus can be seen in the cavernous sinuses (yellow arrows). polymorphs; $35 \%$ lymphocytes), $1.32 \mathrm{~g} / \mathrm{L}$ protein and $3.4 \mathrm{mmol} / \mathrm{L}$ glucose (serum $7.4 \mathrm{mmol} / \mathrm{L}$ ). A right-maxillary sinus trochar washout was performed on day 2 eliciting thick pus, and from this a culture of Streptococcus intermedius (milleri) sensitive to penicillin, but resistant to clarithromycin, was isolated.

Antibiotics empirically commenced on admission were switched to intravenous ceftriaxone with metronidazole when culture results were available and the patient improved over the first week of therapy. Anticoagulation therapy was started in view of the cavernous venous sinus thrombosis. On day 7 , the patient was discharged home to complete a total of 3 weeks of intravenous and 3 weeks of oral antimicrobials under the care of the outpatient parenteral antibiotic therapy team.

\section{Discussion}

This case shows how high-quality imaging complements early recognition of clinical symptoms and successful management of pyogenic bilateral cavernous sinus venous thrombosis (CSVT) secondary to $S$ milleri maxillary sinusitis and otomastoiditis. Although rare, CSVT mortality rate remains as high as $5-10 \%$. Causes of CSVT include infection, malignancy, thrombophilias and pregnancy. Main symptoms include headache, visual loss, ophthalmoplegia, altered consciousness, proptosis and periorbital oedema. Management includes antimicrobial treatment of primary infections (such as sinusitis) and possible complications (including meningitis, surgical drainage and full anticoagulation with heparin). ${ }^{2,3}$ The main clinical differential diagnosis would be osteomyelitis of the petrous temporal bone which can involve cranial nerves, known as Gradenigo's syndrome, but imaging is critical in diagnosis in this case and clearly shows cavernous sinus disease.

\section{Conclusion}

Cavernous sinus venous thrombosis is an uncommon but serious condition which should be considered in the differential diagnosis of patients who present with headache and ophthalmoplegia of recent onset, especially those with a preceding history of localised infection.

\section{References}

1 Ebright JR, Pace MT, Niazi AF. Septic thrombosis of the cavernous sinuses. Arch Intern Med 2001;161:2671-6.

2 Bousser MG, Ferro JM. Cerebral venous thrombosis: an update. Lancet Neurol 2007;6:162-70.

3 Khatri IA, Wasay M. Septic cerebral venous sinus thrombosis. J Neurol Sci 2016:362:221-7.

Address for correspondence: Dr Vanesa Anton-Vazquez, St George's University Hospitals NHS Foundation Trust, Blackshaw Road, London SW17 0QT, UK. Email:vantonva@sgul.ac.uk 